\title{
Meaningful Data Sampling for a Faithful Local Explanation Method
}

\author{
Peyman Rasouli and Ingrid Chieh Yu \\ Department of Informatics, University of Oslo, Norway \\ \{peymanra, ingridcy\}@ifi.uio.no
}

\begin{abstract}
Data sampling has an important role in the majority of local explanation methods. Generating neighborhood samples using either the Gaussian distribution or the distribution of training data is a widely-used procedure in the tabular data case. Generally, this approach has several weaknesses: first, it produces a uniform data which may not represent the actual distribution of samples; second, disregarding the interaction between features tends to create unlikely samples; and third, it may fail to define a compact and diverse locality for the sample being explained. In this paper, we propose a sampling methodology based on observationlevel feature importance to derive more meaningful perturbed samples. To evaluate the efficiency of the proposed approach we applied it to the LIME explanation method. The conducted experiments demonstrate considerable improvements in terms of fidelity and explainability.
\end{abstract}

\section{Introduction}

Explanation methods provide interpretable models for complicated, black box Machine Learning (ML) algorithms such as Random Forests (RF) and Deep Neural Networks [27. These approaches simulate the behaviour of a black box using a simple, interpretable predictor (e.g., linear regression or decision tree) to provide explanations and justifications for the decision being made by the black box. Although black box machine learning models are in demand for automating safety-critical decision systems (e.g., self-driving cars), their applicability is disrupted due to lack of transparency and explainability. Furthermore, black box models are created based on human-made data which may contain bias and prejudices [5. Thus, a resultant learned model may inherited such biases, unconsciously making unfair and incorrect decisions. Creating a faithful explanation method is considered as a first step to elucidate and address these concerns.

The main concern in creating an explanation method is to establish a tradeoff between fidelity and interpretability. By fidelity we mean to which extent the interpretable model is able to accurately imitate a black-box prediction and often is measured in terms the F1-score and Mean Squared Error (MSE) 6. A less-fidelity interpretable model may produce less accurate predictions compared to the black box model. To trust the explanations, the model must therefore be accurate and interpretable simultaneously, as it is unsound to trust explanations 
that are generated based on incorrect predictions. There are a wide range of research works which aim to address the described issue [10/15]14.

Deriving global while accurate explanations of a black box model has been seen as a challenging task 10. In contrast, several local explanation methods have been developed by approximating an interpretable model based on the neighbourhood of a given sample to explain the corresponding prediction of the black box [10]4[12 16]11. They assume that every complex model is linear on a local scale. Local Interpretable Model-agnostic Explanations (LIME), is a fully agnostic method for outcome explanation [10. Given a sample $x$, it generates perturbed samples in the vicinity of $x$, which are weighted based on their proximity to the $x$. LIME maps the perturbed samples to a binary representation for the sake of interpretability. Using a sparse linear model trained on the perturbed samples, LIME explains the reasons behind an outcome in the form of feature importance. In [19, authors presented a Model Explanation System (MES) that explains the prediction of a black box model using a Monte Carlo algorithm. Based on formal requirements, this method constructs a scoring system for finding the best explanations in the form of simple logical rules. MES is both model-agnostic and data-agnostic. Local Rule-based Explanations (LORE) [4, is a model-agnostic approach that addresses black box outcome explanation problem by means of decision rules. It assumes a higher availability of clear and simple decision boundary in the neighbourhood of a data point rather than the whole data space. LORE provides decision rules and counter-factual rules to explain a given sample.

To increase the accountability of the generated explanations, our goal is to achieve an accurate interpretable model similar to the black box model. Specifying the neighbourhood of a given sample is the core task of several perturbationbased explanation methods $10 / 4|12| 16[11$. The locality is defined based on the perturbed data, which later is used for creating an interpretable model. Deriving perturbed samples using either the Gaussian distribution or the distribution of training data is a widely-used procedure. Generally, this approach has several shortcomings; first, it creates a uniform data which may not represent the actual distribution of samples; second, it disregards the interaction between features which tends to generate unlikely data points; and third, it produces a synthetic data set similar to the the training data which may fail to describe a compact locality for a given sample. Besides, relying solely on a distance function for weighting the neighborhood samples is not efficient enough [10. Additive feature attribution methods convert feature values in the original representation to a binary representation for the sake of interpretability 8. This conversion excludes the expressiveness of the data to a great extent and drops the amount of provided information drastically. This causes informative gaps between the original and interpretable representation of the data. The combination of ineffective sampling and information reduction may result in an unrepresentative data set, and accordingly more likely to create unfaithful interpretable models.

Our main contribution is a sampling methodology to produce representative data sets needed for local perturbation-based explanation methods. The 
proposed sampling strategy can be adopted by any model-agnostic explanation method applied on tabular data. Simply put, it exploits the feature importance and the distribution of training data to create a perturbed data set for describing the locality of a given sample. We employ observation-level (not model-level) feature importance which is unique regarding each sample and describes each feature's contribution in the prediction of the black box. The intuition behind the proposed approach is to create a neighborhood data by looking at the locality from both feature space and feature importance perspectives. As each view provides a different insight about the relationship of data points, we leverage them to generate a more expressive and informative data set. In addition we show how our sampling approach can be used in LIME to improve the locality definition in tabular data setting. The sampling in LIME varies in each execution, which means there are instabilities in the generated explanations [9. Through exploiting feature importance, we are bounding the sampling w.r.t the black-box predictions that results in generating more stable explanations. We conducted several experiments on several data sets and compared the results to LIME. The evaluation shows a significant improvement in terms of fidelity and the quality of the generated explanations.

The rest of the paper is organized as follows. In Section 2 we introduce our sampling methodology. In Section 3 we describe the experiments and illustrate the results. Finally, we discuss future work and conclude the paper in Section 4.

\section{Meaningful Data Sampling}

Sampled data points from either the Gaussian distribution or the distribution of training data (e.g., in LIME) may not be representative enough to define a satisfactory locality for the sample being explained. In this paper, we remedy this problem by exploiting feature importance in the sampling process. To this end, we use the sampling function $\phi($.$) defined in Eq. 1$ that takes a sample $x$ as input, the distribution of the training data $\mathcal{D}$, a black box function $f$, and number of perturbed samples $N$; it returns a set of neighborhood samples $\mathcal{Z}$ for creating a local interpretable model,

$$
\mathcal{Z}=\phi(x, \mathcal{D}, f, N)
$$

Our proposed sampling method utilizes observation-level feature importance for creating neighborhood data. To achieve the contribution of each feature in the prediction of a black box model, we exploit the random forests in combination with the treeinterpreter technique [13. The goal is to use the locality information derived from both feature space and target space viewpoints to create a meaningful locality. The proposed method is described in Algorithm 1 .

Random Data Generation. In this step, $N$ perturbed samples, denoted by $\mathcal{Z}^{\prime}$, are drawn from the given distribution $\mathcal{D}$. The proposed method starts with random data generation to achieve perturbed samples with a high degree of diversity and coverage of the feature space. We believe this is an appropriate initialization towards creating a meaningful data set. 


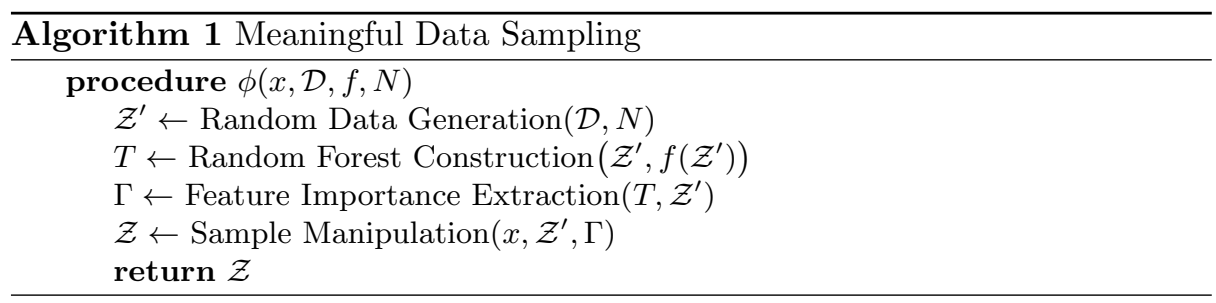

Random Forest Construction. Random Forests (RFs) are widely-used predictive models for both regression and classification tasks 1 . The strength of random forests originates from creating a forest of several random, uncorrelated decision trees and then merging them together in order to gain predictive results. Compared to a decision tree, a random forest adds randomness to the model by means of diverse subsets of samples and features while growing the trees which results in more accuracy and robustness against over-fitting.

Using $\left(\mathcal{Z}^{\prime}, f\left(\mathcal{Z}^{\prime}\right)\right)$ as training data, a random forest classifier, $T$, is created to mime the black box $f$. There are two important reasons for constructing the surrogate model $T$; first, observation-level features' importance of each perturbed sample $z^{\prime} \in \mathcal{Z}^{\prime}$ can be achieved via treeinterpreter; and second, it makes the sampling process to be independent from the type of the black box, therefore, it can be adopted by any local model-agnostic/model-specific explanation method that depends on neighborhood information for explaining an instance. Moreover, it is possible to train $T$ with varied sizes of training data (generated using perturbed samples) and various hyper-parameter settings which allow us to create a robust surrogate model with similar performance to the black box $f$. In this paper, we use standard random forests with default hyper-parameters, leaving the exploration of different configurations to future work.

Feature Importance Extraction. Random forests are typically treated as black box which are infeasible to interpret. Nevertheless, it is possible to turn it into a "white box" using the treeinterpreter. The treeinterpreter is a Python library that uses the underlying trees in a random forest to explain how each feature contributes the end value. Each decision in a tree (or a forest) is followed by a path (or paths) from the root of the tree to the leaf, consisting of a series of decisions, guarded by a particular feature, each of which contribute to the final predictions. Since each decision is guarded by a feature, and the decision either adds or subtracts from the value given in the parent node, the prediction can be defined as the sum of the feature contributions plus the bias. A prediction in a random forest is the average of the prediction of each decision tree. As a result, each prediction is calculated as the average of the bias terms plus the average contribution of each feature.

Model-level feature importance is computed over the entire training data, while treeinterpreter acts on each sample at a time, explaining why a random forest makes a particular decision. For a given sample $x$ and a set of target classes $\mathcal{C}$, feature contributions are represented by vectors $\left\{\gamma_{1}, . ., \gamma_{|\mathcal{C}|}\right\}$ indicating 
the contribution of $x$ to each class. The values of $\gamma$ can be positive, negative, or zero and that all together (plus the bias value) constitute the final prediction. A positive value means that the feature pushes the decision towards the target class, a negative value means that the feature pushes the decision away from the target class, and zero value means that the feature is neutral, so it does not impact the predication.

Using treeinterpreter, feature contribution vectors for all samples in $\mathcal{Z}^{\prime}$ are extracted, forming a feature contribution matrix $\Gamma$. To ease the further operations, $\Gamma$ is discretized using a quantile-based discretization method introduced in [3. The number of bins for each feature is obtained using Sturges's rule [17.

Sample Manipulation. The feature values in $\mathcal{Z}^{\prime}$ and the feature contributions in $\Gamma$, each provides a different type of information about the data. Using importance of features accompanying their values helps to capture the relationship between samples more efficiently. Therefore, we would be able to generate neighborhood instances according to the locality defined by both feature values (explicitly) and feature contributions (implicitly) simultaneously.

In this phase, neighborhood samples are manipulated through comparing the contributions of the given sample, $\Gamma_{x}$, with the contributions of each perturbed sample, $\Gamma_{z^{\prime}}, z^{\prime} \in \mathcal{Z}^{\prime}$ in the following manner: let $\mathcal{C}$ be the set of target classes; for each pair of samples predicted to the same class by $T$, i.e., $\left\{x_{c}, z_{c}^{\prime}\right\}$, where $c \in \mathcal{C}$, their feature contribution vectors for that class are compared; while for a pair of samples predicted to different classes by $T$, i.e., $\left\{x_{c}, z_{c^{\prime}}^{\prime}\right\}$, where $c, c^{\prime} \in \mathcal{C}$ and $c \neq c^{\prime}$, their feature contribution vectors are compared mutually with respect to $c$ and $c^{\prime}$. Since feature importance vector is related to the prediction, we must consider the class of the samples during the comparison. In addition, the contribution of samples to the other classes will not impact this process, which helps to preserve the diversity of the data. It should be noted that we calculate the procedure only for the features of a perturbed sample that have different values than the features of the given sample. Thus, for a given perturbed sample $z^{\prime} \in \mathcal{Z}^{\prime}$, our goal is to identify features with similar contributions to the target class, while their different values has made the $z^{\prime}$ to be located in a distant neighborhood of the given sample $x$. Finally, the perturbed set $\mathcal{Z}$, initialized with $\mathcal{Z}=\mathcal{Z}^{\prime}$, will contain the desired sampled data, as each sample $z \in \mathcal{Z}$ is derived via Eq. (2),

$$
z_{j}= \begin{cases}x_{j}, & \text { if }\left\{\Gamma_{x_{j}}=\Gamma_{z_{j}} \mid x_{j} \neq z_{j}\right\} \\ z_{j}, & \text { otherwise }\end{cases}
$$

where $x$ is the given sample and $j=1, . ., d$ is the dimension of features. The reason behind this replacement is that we assume if a specific feature in a pair of samples has two different values, but similar contributions to a target class, then from the feature importance perspective both samples are similar with respect to that feature; therefore, setting same value for the feature in both samples may not change their predictions significantly, rather making them closer to each other. Doing the mentioned process for all samples in $\mathcal{Z}$ results in a compact and diverse neighborhood of the given sample $x$. 


\section{Experiments and Discussions}

In this section, the proposed method is evaluated with respect to several classification data sets and black box models. To restate, the main goal of this work is to improve the fidelity, showing to which extent the interpretable model $g$ is able to accurately imitate the black box model $f$. We adopted linear regressions and decision trees as interpretable models simultaneously to offer a better insight about the fedility of the explanation method.

Experiments and Benchmarks. The proposed sampling method has been developed in Python programming language and experiments were run on a system with Intel Core i7-8650U processor and 32GB of memory. We used scikit-learn libraries for implementing the machine learning and data mining algorithms. We applied our sampling method on LIME and benchmarked it against LIME with its original sampling strategy. As a convention, we use S-LIME to refer the LIME that is armed with our sampling technique. The evaluation results are reported in three parts: $(i)$ fidelity comparison, $(i i)$ neighborhood analysis, and (iii) explanation comparison.

Experimental Setup. In the experiments, four tabular classification data sets including recidivism 1 , adult, german credit, and red wine quality ${ }^{2}$ were used. Each data set was split to $85 \%$ train set and $15 \%$ test set. A multi-class AdaBoost classifier [20 (AdB) and a Random Forest classifier (RF) each with 300 estimators were employed as black box models. The $50 \%$ of test set were used to quantify the fidelity of the explanation methods. The number of neighborhood samples was set to $N=1000$. Each sample was explained using $K=\{2, . ., 5\}$ features and the results of $K$ with the highest performance was considered.

The following properties are used for evaluating the performance of interpretable models, i.e., decision tree $g^{D}$ and liner regression $g^{L}$, in miming the local behavior of the black box $f$. The notions $y$ and $Y$ are the ground-truth labels and $\hat{y}$ and $\hat{Y}$ are the predicted labels of a sample and the entire data set:

- $\boldsymbol{h i t}_{\boldsymbol{g}^{\boldsymbol{D}}}(y, \hat{y}) \in[0,1]$. It compares the prediction of $g^{D}$ and black box $f$ on the sample of interest $x$ using F1-score.

- $\boldsymbol{h i t}_{\boldsymbol{g}^{\mathbf{L}}}(y, \hat{y}) \in[0,1]$. It compares the prediction of $g^{L}$ and black box $f$ on the sample of interest $x$ using Mean Absolute Error (MAE).

- $\boldsymbol{f i d e l i t y}_{\boldsymbol{g}^{D}}(Y, \hat{Y}) \in[0,1]$. It compares the predictions of $g^{D}$ and black box $f$ on the training samples $\mathcal{Z}$ using F1-score.

- $\boldsymbol{f i d e l i t y}_{\boldsymbol{g}^{L}}(Y, \hat{Y}) \in[0,1]$. It compares the predictions of $g^{L}$ and black box $f$ on the training samples $\mathcal{Z}$ using R2-score.

The $\boldsymbol{f i d e l i t}_{\boldsymbol{g}^{D}}$ and $\boldsymbol{f i d e l i t} \boldsymbol{y}_{\boldsymbol{g}^{L}}$ metrics describe how good are the interpretable models at imitating the black box model. The desired values for $\boldsymbol{h i t}_{\boldsymbol{g}^{\boldsymbol{D}}}, \boldsymbol{f i d e l i t}_{\boldsymbol{g}^{\boldsymbol{D}}}$, and $\boldsymbol{f i d e l i t y}_{\boldsymbol{g}^{L}}$ are 1.0 , while it is 0.0 for $\boldsymbol{h i t}_{\boldsymbol{g}^{\boldsymbol{L}}}$. We executed 10 iterations of the experiment for each data set and black box model, hence the average and standard deviation of the results are reported in the following.

\footnotetext{
${ }^{1}$ Data set is available at: https://www.kaggle.com/danofer/compass

2 Data sets are available at: https://archive.ics.uci.edu/ml/datasets/
} 
Table 1. LIME vs S-LIME: comparison of fidelity for RF black box model.

\begin{tabular}{c|c|c|c|c|c}
\hline Data set & Method & hit $_{g^{D}}$ & hit $_{g^{L}}$ & fidelity $_{g}{ }^{D}$ & fidelity $_{g}{ }^{L}$ \\
\hline \multirow{2}{*}{ adult } & LIME & $0.845 \pm 0.052$ & $0.126 \pm 0.007$ & $0.910 \pm 0.008$ & $0.426 \pm 0.013$ \\
& S-LIME & $\mathbf{0 . 9 1 5} \pm \mathbf{0 . 0 2 6}$ & $\mathbf{0 . 1 0 5} \pm \mathbf{0 . 0 1 1}$ & $\mathbf{0 . 9 1 4} \pm \mathbf{0 . 0 0 8}$ & $\mathbf{0 . 4 6 3} \pm \mathbf{0 . 0 1 3}$ \\
\hline \multirow{2}{*}{ german } & LIME & $0.864 \pm 0.030$ & $0.103 \pm 0.006$ & $0.822 \pm 0.008$ & $0.347 \pm 0.020$ \\
& S-LIME & $\mathbf{0 . 8 8 6} \pm \mathbf{0 . 0 4 4}$ & $\mathbf{0 . 0 9 3} \pm \mathbf{0 . 0 0 4}$ & $\mathbf{0 . 8 3 4} \pm \mathbf{0 . 0 0 7}$ & $\mathbf{0 . 3 7 8} \pm \mathbf{0 . 0 2 5}$ \\
\hline \multirow{2}{*}{ recidivism } & LIME & $1.000 \pm 0.000$ & $0.027 \pm 0.002$ & $0.931 \pm 0.003$ & $0.791 \pm 0.010$ \\
& S-LIME & $\mathbf{1 . 0 0 0} \pm \mathbf{0 . 0 0 0}$ & $\mathbf{0 . 0 2 3} \pm \mathbf{0 . 0 0 2}$ & $\mathbf{0 . 9 4 8} \pm \mathbf{0 . 0 0 3}$ & $\mathbf{0 . 8 3 1} \pm \mathbf{0 . 0 0 8}$ \\
\hline \multirow{2}{*}{ red wine } & LIME & $\mathbf{0 . 8 5 4} \pm \mathbf{0 . 0 2 3}$ & $0.202 \pm 0.012$ & $0.658 \pm 0.005$ & $0.216 \pm 0.008$ \\
& S-LIME & $0.845 \pm 0.019$ & $\mathbf{0 . 1 8 4} \pm \mathbf{0 . 0 0 9}$ & $\mathbf{0 . 6 8 5} \pm \mathbf{0 . 0 0 5}$ & $\mathbf{0 . 2 6 2} \pm \mathbf{0 . 0 0 9}$
\end{tabular}

Fidelity Comparison. Tables 1 and 2 report the statistical results for $\mathbf{R F}$ and AdB black box models, respectively. They exhibit overall improvement of S-LIME over LIME concerning all data sets and black box models. The individual sample evaluation metrics, i.e., $\boldsymbol{h i t}_{\boldsymbol{g}^{\boldsymbol{D}}}$ and $\boldsymbol{h i t}_{\boldsymbol{g}^{\mathbf{L}}}$, are considerably important in measuring the fidelity of the intepretable models; according to the listed values for these properties, S-LIME classified a higher proportion of samples correctly compared to LIME. Especially, for adult and red wine quality data sets the improvements are more prominent. In addition, fidelity $\boldsymbol{g}^{\boldsymbol{D}}$ and $\boldsymbol{f i d e l i t}_{\boldsymbol{g}^{\boldsymbol{L}}}$ show more accurate predictions of the neighborhood samples in S-LIME; they indicate that the explanations were derived from faithful local interpretable models.

Table 2. LIME vs S-LIME: comparison of fidelity for AdB black box model.

\begin{tabular}{c|c|c|c|c|c}
\hline Data set & Method & hit $_{g^{D}}$ & hit $_{g^{L}}$ & fidelity $_{g} D$ & fidelity $_{g}{ }^{L}$ \\
\hline \multirow{2}{*}{ adult } & LIME & $0.840 \pm 0.055$ & $0.008 \pm 0.001$ & $0.853 \pm 0.020$ & $0.238 \pm 0.040$ \\
& S-LIME & $\mathbf{0 . 8 8 9} \pm \mathbf{0 . 0 4 1}$ & $\mathbf{0 . 0 0 6} \pm \mathbf{0 . 0 0 1}$ & $\mathbf{0 . 8 5 9} \pm \mathbf{0 . 0 1 9}$ & $\mathbf{0 . 2 5 9} \pm \mathbf{0 . 0 4 1}$ \\
\hline \multirow{2}{*}{ german } & LIME & $0.816 \pm 0.045$ & $0.002 \pm 0.000$ & $0.730 \pm 0.012$ & $0.075 \pm 0.027$ \\
& S-LIME & $\mathbf{0 . 8 5 4} \pm \mathbf{0 . 0 4 2}$ & $\mathbf{0 . 0 0 2} \pm \mathbf{0 . 0 0 1}$ & $\mathbf{0 . 7 4 9} \pm \mathbf{0 . 0 1 0}$ & $\mathbf{0 . 0 8 5} \pm \mathbf{0 . 0 3 4}$ \\
\hline \multirow{2}{*}{ recidivism } & LIME & $0.996 \pm 0.003$ & $0.002 \pm 0.000$ & $0.927 \pm 0.005$ & $0.738 \pm 0.010$ \\
& S-LIME & $\mathbf{0 . 9 9 6} \pm \mathbf{0 . 0 0 3}$ & $\mathbf{0 . 0 0 1} \pm \mathbf{0 . 0 0 0}$ & $\mathbf{0 . 9 4 5} \pm \mathbf{0 . 0 0 3}$ & $\mathbf{0 . 7 8 4} \pm \mathbf{0 . 0 0 8}$ \\
\hline \multirow{2}{*}{ red wine } & LIME & $0.894 \pm 0.060$ & $0.020 \pm 0.011$ & $0.572 \pm 0.083$ & $0.047 \pm 0.032$ \\
& S-LIME & $\mathbf{0 . 9 7 0} \pm \mathbf{0 . 0 1 5}$ & $\mathbf{0 . 0 1 8} \pm \mathbf{0 . 0 1 1}$ & $\mathbf{0 . 6 2 0} \pm \mathbf{0 . 0 8 7}$ & $\mathbf{0 . 0 5 2} \pm \mathbf{0 . 0 3 6}$ \\
\hline
\end{tabular}

Fig. 1(a) illustrates the number of interpretable components/features represented to the user. It reveals the required number of features for explaining a sample, i.e., $\boldsymbol{k}$, varies regarding the data sets and black box models; however, both methods have shown that samples can be explained with 3 or 4 features roughly. Referring to Fig. 1(b) the only downside of S-LIME is a negligible extra time required for explaining a sample that is not remarkable compared to the faithfulness of explanations. It is worth noting that choosing a proper neighborhood size $N$ as well as finding suitable hyper-parameters for $T$ can further improve the performance of the explanation method. 


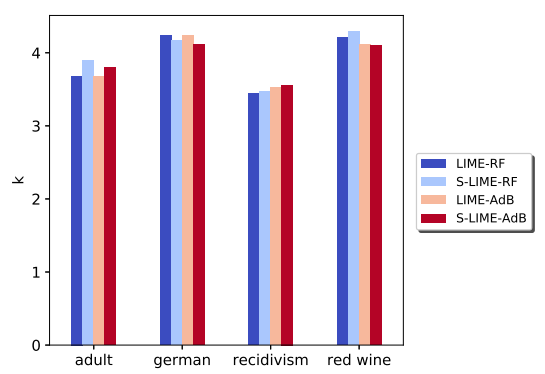

(a) Explanation size

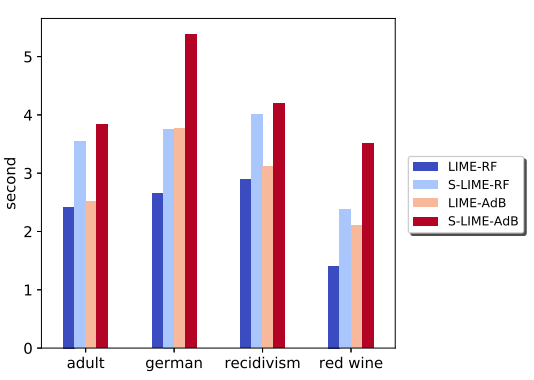

(b) Execution time

Fig. 1. LIME vs S-LIME: comparison of explanation size and execution time.

Neighborhood Analysis. We analyzed the effect of the proposed sampling method in the formation of the locality for the sample being explained. As mentioned earlier, compactness and diversity are two important factors in generating neighborhood samples. According to our conducted experiments, we observed that S-LIME generates a higher number of data points in the proximity of the given sample. One example of this observation is illustrated in Fig. 2, which demonstrates the distribution of samples in the vicinity of a given sample from recidivism data set. For visualizing the data points in a $2-\mathrm{D}$ space, Principal Component Analysis (PCA) [18] is used to reduce the feature dimension.
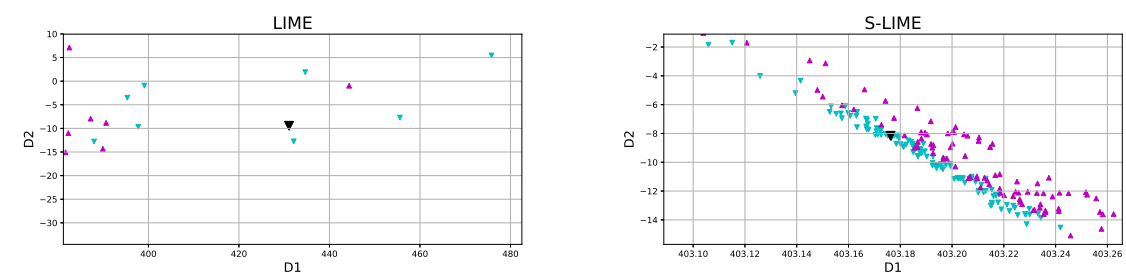

Fig. 2. Neighborhoods of LIME (left) and S-LIME (right). The ' $\boldsymbol{\nabla}$ ' denotes the sample being explained.

According to Tables 1 and 2, our devised sampling improves the fidelity of the explanation method significantly without creating exceedingly similar samples or an imbalanced data set. Based on analysis results listed in Table 3 , the proposed method, on average, makes a minor number of features, i.e., $\boldsymbol{n}_{\text {features }}$, in each neighborhood data point similar to the given sample. Moreover, the percentage of instances that are predicted differently to the opposite class, i.e., $\boldsymbol{n}_{\boldsymbol{p r e d s}}$, is about $1 \%$. As a result, the sampling procedure manipulates the features that are 
less prone to change the prediction of the sample. Therefore, by adopting the proposed sampling, we transform a randomly generated data to a meaningful one (with respect to the given sample) which is highly diverse and representative.

Table 3. Sample manipulation analysis.

\begin{tabular}{c|c|c|c|c}
\hline Data set & \multicolumn{2}{|c|}{ adult } & \multicolumn{2}{c}{ german } \\
\hline Black Box & $n_{\text {features }}$ & $n_{\text {preds }}$ & $n_{\text {features }}$ & $n_{\text {preds }}$ \\
\hline RF & $0.682 \pm 0.029$ & $0.7 \% \pm 0.1 \%$ & $1.267 \pm 0.091$ & $1.1 \% \pm 0.1 \%$ \\
AdB & $0.563 \pm 0.023$ & $0.7 \% \pm 0.1 \%$ & $0.942 \pm 0.054$ & $1.8 \% \pm 0.4 \%$ \\
\hline
\end{tabular}

Explanation Comparison. In Fig. 3, generated explanations using explanation methods, i.e., LIME and S-LIME, are shown for a sample from adult data set. Above each explanation, the predicted class and probability by the black box $f$ as well as the predicted class by decision tree $g^{D}$ and the response value predicted by linear regression $g^{L}$ are stated. An explanation is directly related to the prediction of the interpretable model, hence it is vital to establish the fidelity of its prediction before one count on the provided explanation. Fig. 3 reveals that S-LIME predicted the sample to the correct class with a high probability while LIME classifies the sample incorrectly. To make sure about the stability of predictions and corresponding explanations, we repeated the experiments multiple times; LIME experienced some fluctuation while S-LIME produced more uniform results in terms of prediction and explanation.
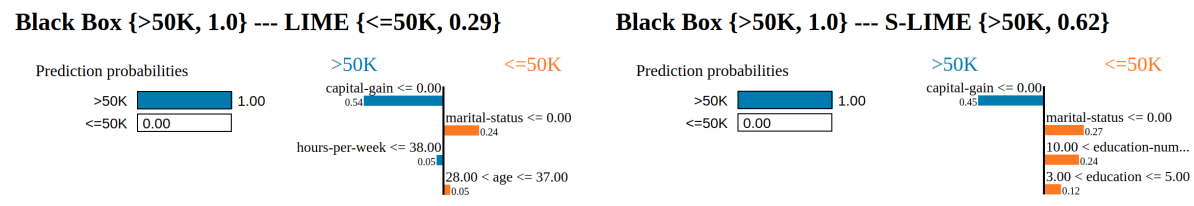

Fig. 3. Explanations of LIME (left) and S-LIME (right).

\section{Conclusion and Future Work}

In this paper, we introduced a meaningful data sampling technique applicable to local explanation methods. It exploits the similarity of feature values and feature importance viewpoints simultaneously to create a diverse and compact locality for the sample being explained. The methodology can be integrated into several local explanation methods for achieving meaningful and representative neighborhood samples. We demonstrated its applicability by applying it to the state-of-the-art explanation method, LIME. The obtained results are promising 
in terms of fidelity and explainability. In future work, we will study the effect of the proposed approach on rule-based explanation methods. Furthermore, its applicability on a real world use-case will be investigated.

\section{References}

1. G. Biau and E. Scornet. A random forest guided tour. Test, 25(2):197-227, 2016.

2. L. Breiman. Random Forests. Machine Learning, 45:5-32, 2001.

3. J. Dougherty, R. Kohavi, and M. Sahami. Supervised and Unsupervised Discretization of Continuous Features. Proc. Machine Learning, pages 194-202, 1995.

4. R. Guidotti, A. Monreale, S. Ruggieri, D. Pedreschi, F. Turini, and F. Giannotti. Local rule-based explanations of black box decision systems. CoRR, 2018.

5. R. Guidotti, A. Monreale, S. Ruggieri, F. Turini, F. Giannotti, and D. Pedreschi. A survey of methods for explaining black box models. ACM computing surveys, 51(5):93, 2018.

6. J. Han, M. Kamber, and J. Pei. Data mining : concepts and techniques. Elsevier Science, 2011.

7. A. Krizhevsky, I. Sutskever, and G. E. Hinton. ImageNet Classification with Deep Convolutional Neural Networks. In Advances in Neural Information Processing Systems, pages 1097-1105. Curran Associates, Inc., 2012.

8. S. M. Lundberg and S.-I. Lee. A unified approach to interpreting model predictions. In Advances in Neural Information Processing Systems, pages 4765-4774, 2017.

9. C. Molnar. Interpretable Machine Learning. 2019.

10. M. T. Ribeiro, S. Singh, and C. Guestrin. Why Should I Trust You? Explaining the Predictions of Any Classifier. Proc. of the 22nd ACM SIGKDD Int. Conf. on Knowledge Discovery and Data Mining, pages 1135-1144, 2016.

11. M. Robnik-Šikonja and M. Bohanec. Perturbation-Based Explanations of Prediction Models. In Human and Machine Learning, pages 159-175. Springer, 2018.

12. M. Robnik-Sikonja and I. Kononenko. Explaining Classifications For Individual Instances. IEEE TKDE, 20(5):589-600, 2008.

13. A. Saabas. treeinterpreter library, 2019.

14. A. Shrikumar, P. Greenside, and A. Kundaje. Learning important features through propagating activation differences. In Proc. of the 34th Int. Conf. on Machine Learning, pages 3145-3153, 2017.

15. E. Strumbelj and I. Kononenko. Explaining prediction models and individual predictions with feature contributions. Knowl. Inf. Syst., 41:647-665, 2014.

16. E. Štrumbelj, I. Kononenko, and M. Robnik Šikonja. Explaining instance classifications with interactions of subsets of feature values. Data $\mathscr{E}$ Knowledge Engineering, 68(10):886-904, 2009.

17. H. A. Sturges. The Choice of a Class Interval. Journal of the American Statistical Association, 21(153):65-66, 1926.

18. M. E. Tipping and C. M. Bishop. Probabilistic principal component analysis. Journal of the Royal Statistical Society: Series B, 61(3):611-622, 1999.

19. R. Turner. A model explanation system. In IEEE 26th Int. Work. on Machine Learning for Signal Processing, pages 1-6, 2016.

20. J. Zhu, H. Zou, S. Rosset, and T. Hastie. Multi-class AdaBoost *. Statistics and Its Interface, 2:349-360, 2009. 